\title{
The referential convergence of gene concepts based on classical and molecular analyses
}

\author{
By: Tudor Baetu
}

Kenneth Waters and Marcel Weber argue that the joint use of distinct gene concepts and the transfer of knowledge between classical and molecular analyses in contemporary scientific practice is possible because classical and molecular concepts of the gene refer to overlapping chromosomal segments and the DNA sequences associated with these segments. However, while pointing in the direction of coreference, both authors also agree that there is a considerable divergence between the actual sequences that count as genes in classical genetics and molecular biology. This paper combines a philosophical and historical analysis of the discovery and use of complementation assays by the Morgan school and Seymour Benzer in order to show that the referents of classical and molecular gene concepts are coextensive to a much higher degree than admitted by Waters and Weber, and therefore coreference can provide a satisfactory account of the high level of integration between classical genetics and molecular biology. In particular, I argue that the functional units/cistrons identified by classical techniques overlap with functional elements entering the composition of molecular transcription units, and that the precision of this overlap can be improved by conducting further experimentation. 